\title{
Alpha-naphthyl Acetate Esterase
}

National Cancer Institute

\section{Source}

National Cancer Institute. Alpha-naphthyl Acetate Esterase. NCI Thesaurus. Code C89858.

A selective cytochemical monocyte marker. 